OPEN

SUBJECT AREAS:

PHASE TRANSITIONS

AND CRITICAL

PHENOMENA

CHEMICAL PHYSICS

Received

9 July 2014

Accepted

17 September 2014

Published

16 October 2014

Correspondence and requests for materials should be addressed to L.M.X. (limei.xu@pku. edu.cn)

\title{
Electrostatic field-exposed water in nanotube at constant axial pressure
}

\author{
Yuchi He' ${ }^{1}$ Gang Sun', Kenichiro Koga ${ }^{2} \&$ Limei Xu ${ }^{1,3}$ \\ ${ }^{1}$ International Center for Quantum Materials and School of Physics, Peking University, ${ }^{2}$ Department of Chemistry, Okayama \\ University, Tsushima-Naka 3-1-1, Okayama 700-8530, Japan, ${ }^{3}$ Collaborative Innovation Center of Quantum Matter, Beijing, \\ China.
}

Water confined within nanoscale geometries under external field has many interesting properties which is very important for its application in biological processes and engineering. Using molecular dynamics simulations, we investigate the effect of external fields on polarization and structure as well as phase transformations of water confined within carbon nanotubes. We find that dipoles of water molecules tend to align along external field in nanoscale cylindrical confinement. Such alignment directly leads to the longitudinal electrostriction and cross-sectional dilation of water in nanotube. It also influences the stability of ice structures. As the electrostatic field strengthens, the confined water undergoes phase transitions from a prism structure to a helical one to a single chain as the electrostatic field strengthens. These results imply a rich phase diagram of the confined water due to the presence of external electriostatic field, which can be of importance for the industrial applications in nanopores.

W ater exists in nanopores of cylindrical geometry such as the ion channels ${ }^{1,2}$, porous silicas ${ }^{3,4}$, and carbon nanotubes ${ }^{5-9}$. Such nanoconfined water is important not only for biological processes but also for industrial applications including filtration and sorption, petrochemical refining, nuclear waste disposal, and heterogeneous catalysis ${ }^{10,11}$. For example, water in carbon nanotubes has high flow rate, high proton conductivity, and highly selective transport of solutes and water $^{7-9}$, and these properties may be utilized for designing nanofluidic and molecular separation devices or fuel cells ${ }^{12}$. The presence of such properties is mainly due to the remarkable ability of water to form a variety of hydrogen-bonded arrangements in cylindrically confined geometry ${ }^{5,6}$. However, the structural arrangement of hydrogen bonds in confined water can be quite sensitive to nonthermal variables, e.g., size of nanopores, chemical and physical properties of pore surfaces, and electrostatic fields due to the presence of ions in membranes and ion channels.

The effects of electrostatic fields on water have been extensively studied ${ }^{13-19}$. Computer simulation studies of water in nanotubes have shown that electrostatic field of magnitude $\sim 1 \mathrm{~V} / \mathrm{nm}$ is enough to alter the wetting property of carbon nanotubes ${ }^{16}$ and leads to electrostriction as well as structure transitions, e.g., a transition from polygonal prism ice to helical structure ${ }^{20}$. These results demonstrate that the electrostatic field can dramatically change the properties of water by disrupting or altering the arrangements of water molecules. However, how these changes are related to the polarization of water is not well understood. Additionally, due to the difficulty in either experimentally controlling of the electrostatic field or measuring the nanoscale structures, the experimental studies on nanoconfined water under strong electrostatic fields $(>0.1 \mathrm{~V} / \mathrm{nm})^{21-25}$ are lacking. Hence, the computer simulation of such systems serves as a valuable tool for understanding the behaviors of water under the electrostatic field.

In this work, using molecular dynamics simulations, we systematically study the effect of external fields on the properties of water confined in carbon nanotubes. We investigate external field effect on the polarization of water and structure transformation. The manuscript is arranged as follows. The simulation details are described in the Methods section, the effect of the uniaxial field on water confined in nanotubes is presented in Results section, and Discussions as well as a biref summary are given in the end.

\section{Results}

Effect of external electric field on polarization. Various properties of a liquid of polar molecules change as the uniaxial electrostatic field is applied. Those changes are mainly due to the change in orientation polarization, $\cos \theta$, where $\theta$ is the angle between the dipole of water molecule and the uniaxial external field, $E_{z}$, applied along the $z-$ axis of the nanotube with radius $R$. Figure 1 shows the average polarization, $\left\langle\cos \theta>\right.$, as a function of $E_{z}$ for water confined in the nanotube with $R=5.55 \AA$ at $T=320 \mathrm{~K}$. The polarization increases as $E_{z}$ increases, and becomes 


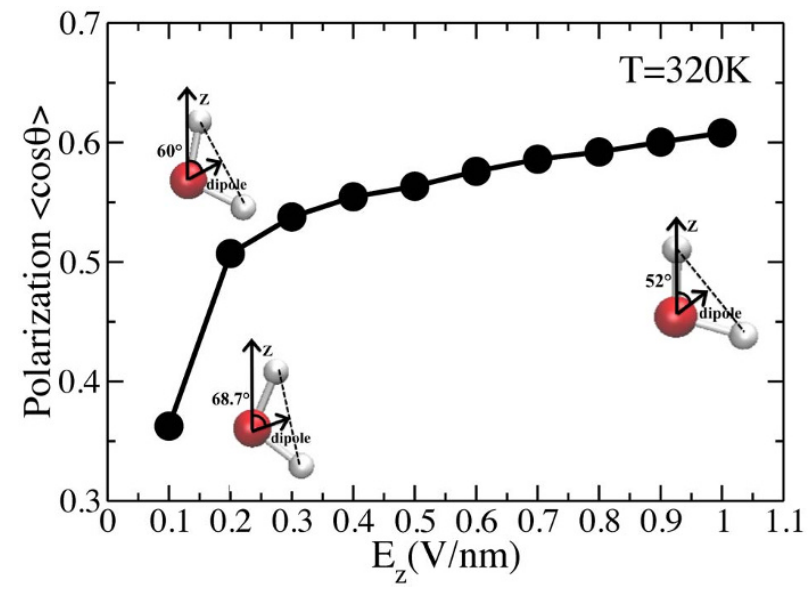

Figure $1 \mid$ The average polarization, $<\cos \theta>$, of water molecules in the SWCN with radius $R=5.55 \AA$ as a function of uniaxial electric field, $E_{z}$ for $T=320 \mathrm{~K}$ and $P_{z}=1 \mathrm{~atm}$. As $E_{z}$ increases, $<\cos \theta>$ increases before it saturates to $\sim 0.615$. The system also becomes more structured as $E_{z}$ increases, e.g., at $E_{z}=1 \mathrm{~V} / \mathrm{nm}, \cos \theta=0.615$, one of the hydrogen bonds of each water molecule is nearly parallel to the $z$-direction.

more or less saturated to a value $\sim 0.615$ at $E_{z}=1 \mathrm{~V} / \mathrm{nm}$. This corresponds to a gradually lineup of dipoles along the nanotube, from $\theta \sim 70^{\circ}$ for $E_{z}=0.1 \mathrm{~V} / \mathrm{nm}$ to $\theta \sim 52^{\circ}$ (corresponding to half of the $\mathrm{HOH}$ angle of water molecule, $104^{\circ}$, i.e. one of the hydrogen bonds of the water molecule is parallel to the $z$ direction) for $E_{z}>1 \mathrm{~V} / \mathrm{nm}$. This indicates that the system becomes more ordered with increasing $E_{z}$, e.g., one of the hydrogen bonds of the water molecule becomes almost parallel to the $z$-direction, as shown in the inset of Figure 1.

Compared to that of bulk water, the polarization of water confined in the SWCNT is larger (see Table I). In addition, the distributions of polarization of water molecules are different [Figure 2]. For instance, at $E_{z}=0.1 \mathrm{~V} / \mathrm{nm}$, the distribution of the polarization of bulk water shows a linear behavior over the range $0.1<\cos \theta<0.6$, while it follows an exponential behavior over the same range for water confined in the SWCNT.

Electrostriction. The presence of electrostatic field may change the properties of water. For instance, applying electrostatic field or adding ions to water confined in ion channels and carbon nanotubes leads to the increase of water density, also called "electrostriction"14,16,26-28. Such phenomenon is generally found when field exposed water is held in equilibrium with a nonperturbed reservoir ${ }^{15,27,29}$. In bulk and two dimensional confined case, maintaining pressure inside the field-exposed water unchanged may require expansion ${ }^{15,30,31}$. Interestingly, in the present system, as $E_{z}$ increases, the linear density of water along the axis of the nanotube increases, while the average distance of water molecules (the oxygen atoms) to the tube axis increases [Figure 3], which is different from previous results mentioned above. In addition, the structure of the confined liquid, determined by the number of hydrogen bonds per molecule shown in Table II, becomes more ordered as $E_{z}$ increases.

Table I Average polarization $<\cos \theta>$ in confined and bulk water at $\mathrm{T}=320 \mathrm{~K}$

\begin{tabular}{lcc}
$E(\mathrm{~V} / \mathrm{nm})$ & $<\cos \theta>$ (bulk) & $<\cos \theta>$ (confined) \\
\hline 0.1 & 0.111 & 0.363 \\
0.2 & 0.191 & 0.507 \\
0.3 & 0.245 & 0.538 \\
\hline
\end{tabular}

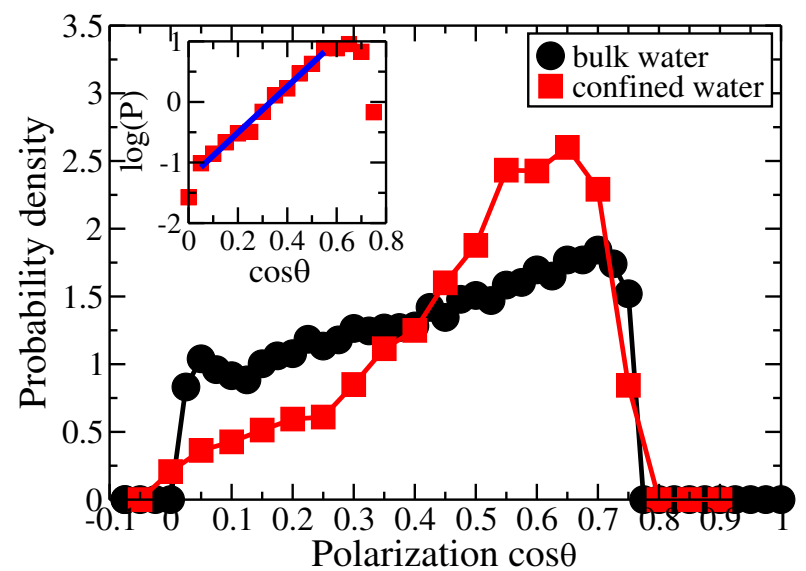

Figure $2 \mid$ Distribution of polarizations for water molecules at $T=320 \mathrm{~K}$ and $E_{z}=0.1 \mathrm{~V} / \mathrm{nm}$. Compared to that for bulk water, the distribution for water confined within $R=5.55 \AA$ is truncated at $\cos \theta=0$ and $\cos \theta \approx 0.75$. The inset shows that the polarization follows Boltzmann distribution for $0<E_{z}<5.5 \mathrm{~V} / \mathrm{nm}$, indicated by a linear relation of $\log P$ with $\cos \theta$.

Stability of ordered States. In the case of zero-field $\left(E_{z}=0\right)$, Koga et al. ${ }^{5,6}$ studied the phase diagram of water confined in nanotube with $R=5.55 \AA$. They found that the ordered structures, such as square, pentagonal, and hexagonal prisms, are stable at room temperature, which was also verified by experiment ${ }^{32-34}$. If the ice structures are further characterized by the $\mathrm{O}-\mathrm{H}$ bond direction (from oxygen atom to hydrogen atom) along each chain of water molecules parallel to the tube axis, one ordered ice structure may contain several different substructures, e.g., there exist four types of square prism structures (see Figure 4).

The square prism structure has four chains of water molecules as shown in Figure $4(\mathrm{a}-\mathrm{d})$. The coupling among these chains is antiferroelectric, so the Hamiltonian of the system can be written as

$$
\begin{aligned}
H= & -J_{1}\left(p_{1} p_{2}+p_{2} p_{3}+p_{3} p_{4}+p_{4} p_{1}\right)-J_{2}\left(p_{1} p_{3}+p_{2} p_{4}\right) \\
& -E_{z}\left(p_{1}+p_{2}+p_{3}+p_{4}\right),
\end{aligned}
$$

where $p_{i}= \pm 1$, with +1 parallel to $E_{z}$ and -1 antiparallel to $E_{z}$, and $J_{i}$ is the coupling between two water chains with $J_{1}$ the neighboring coupling constant and $J_{2}$ the diagonal coupling constant, $J_{1}<J_{2}<0$. According to Eq. 1, the energies of the four structures shown in Figure 4 are: $4 J_{1}-2 J_{2}$ for structure A, $2 J_{2}$ for structure B, 0 for structure $\mathrm{C}$, and $-4 J_{1}-2 J_{2}$ for structure $\mathrm{D}$, respectively. Therefore, structure $\mathrm{A}$ has the lowest energy at $E_{z}=0$. In the pres-

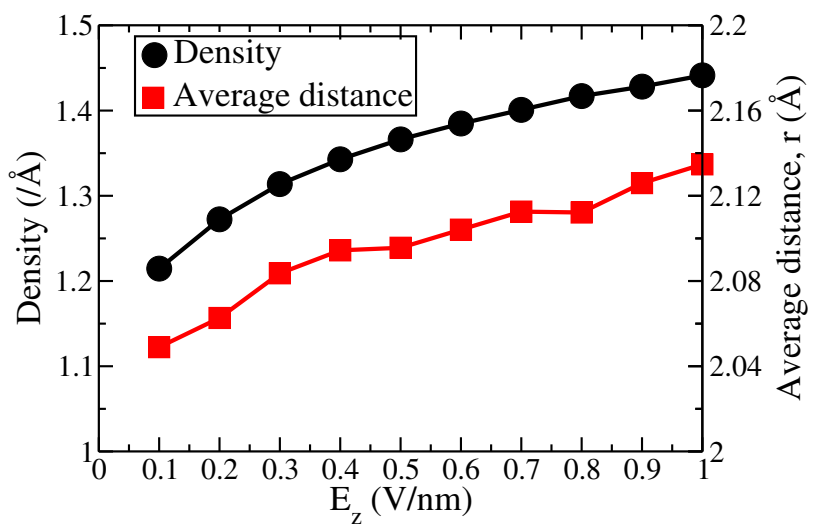

Figure 3 Effect of the uniaxia electric field on the structure of the confined water. The linear density and the average distance of water molecule to the SWCNT axis increases with the external field. 


\begin{tabular}{|lc|}
$\begin{array}{l}\text { Table II } \mid \text { Average number of hydrogen bonds per water molecule } \\
\text { in nanotube at } \mathrm{T}=320 \mathrm{~K}\end{array}$ \\
$\mathrm{E}_{\mathrm{z}}$ & Hydrogen bonds \\
\hline 0.1 & 2.506 \\
0.5 & 2.625 \\
0.8 & 2.916 \\
1.0 & 2.960 \\
\hline
\end{tabular}

ence of external field $E_{z}$, the relative stability of different structures may change. In this case, the energy of structure $D, H=-4 J_{1}-2 J_{2}$ $-4 E_{z}$, is now the lowest. When the electrostatic field decreases from $E_{z}=0.2 \mathrm{~V} / \mathrm{nm}$, we find that the structure $D$ still exists at $E_{z}=0$, indicating the existence of hysteresis in structure $D$.

Structure transformations. When all $\mathrm{O}-\mathrm{H}$ bonds on the edge of ice prism align parallel to $E_{z}$, further increase in $E_{z}$ leads to phase transformations among various ordered structures. Such structure transformations of confined water in the presence of $E_{z}$ can be determined by the changes in average polarization, $<\cos \theta>$. As can be seen from Figure 5 , at $T=250 \mathrm{~K}$, water in nanotube with $R=6.24 \AA$ is stable in hexagonal prism structure, denoted by $(6,0)$, for $0<E_{z}<0.9 \mathrm{~V} / \mathrm{nm}$, in helix structure denoted by $(6,1)$ for $0.9 \mathrm{~V} /$ $\mathrm{nm}<E_{z}<1.12 \mathrm{~V} / \mathrm{nm}$, and in a single chain inside structure denoted 6-shell-chain $(6+1)$ for $1.12 \mathrm{~V} / \mathrm{nm}<E_{z}<2 \mathrm{~V} / \mathrm{nm}$. We note that the first index " 6 " represents the number of water chains in the ice structure, and the second index, "0" or " 1 ", represents the degree of helical structure (For more details, see Ref. [6]).

For different size of carbon nanotubes, the transformations among the ordered structures are also different. For instance, for $R=5.55 \AA$ and at $T=260 \mathrm{~K}$, five types of ordered structure, square prism, pentagonal prism, $(5,1)$ structure, $(5,2)$ structure and a combination of $(5,2)$ shell and a single chain inside denoted by 5 -shell-chain structure $(5+1)$, are observed in the range of $0<E_{z}<8 \mathrm{~V} / \mathrm{nm}$ at $T=260 \mathrm{~K}$ [Fig. 6]. The stability of different structures changes as $E_{z}$ varies. The system is stable in square prism structure for $0<E_{z}<$

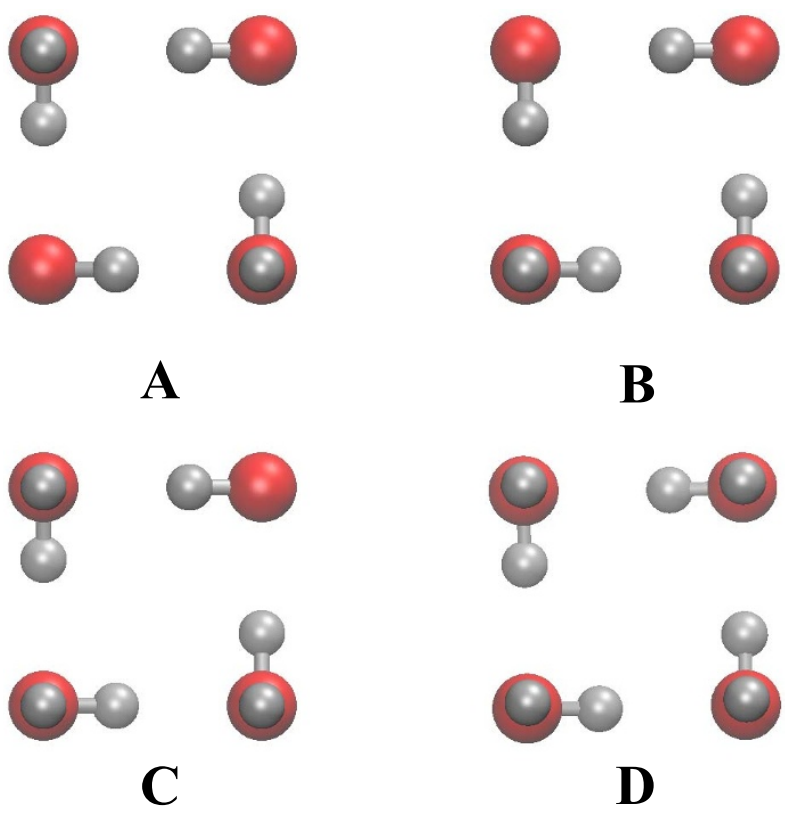

Figure 4 | The skematics of the cross section structures of square prism structures. There are four types of square ice tube. According to Eq. 1, in the absence of external field, the energies of these structures follows that $E_{A}$ $<E_{B}<E_{C}<E_{D}$, while in the presence of large enough external electric field, the energy of the structure $\mathrm{D}$ has the lowest energy.

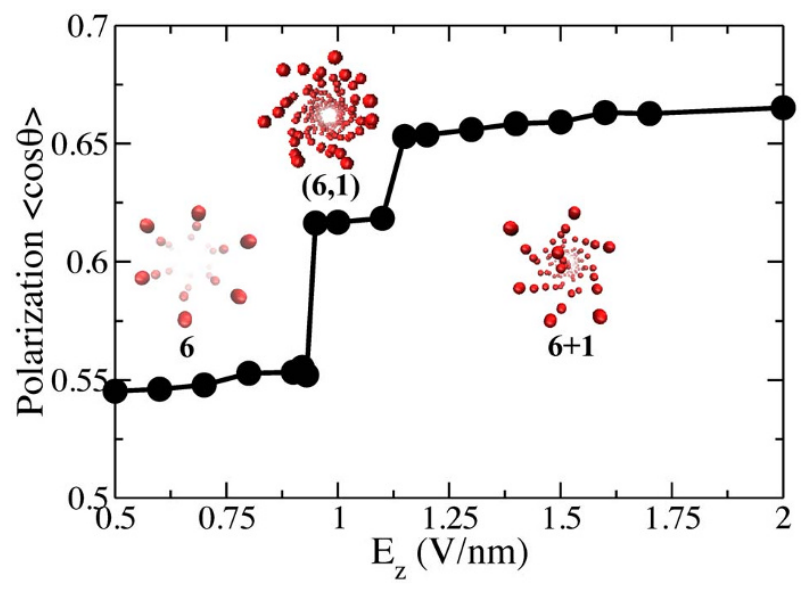

Figure $5 \mid$ Polarization as function of $E_{z}$ for $R=6.24 \AA$ in the presence of the external electric field. The structural changes can be clearly seen from the sharp changes in polarization as $E_{z}$ increases. For instance, the system is hexagonal structure $(6,0)$ for $0<E_{z}<0.9 \mathrm{~V} / \mathrm{nm}$, helical structure $(6,1)$ for $0.9 \mathrm{~V} / \mathrm{nm}<E_{z}<1.12 \mathrm{~V} / \mathrm{nm}$, and 6-shell-chain structure $(6+1)$ for $E_{z}>1.12 \mathrm{~V} / \mathrm{nm}$.

$0.5 \mathrm{~V} / \mathrm{nm}$, in pentagonal prism structure denoted by $(5,0)$ for $0.5 \mathrm{~V} /$ $\mathrm{nm}<E_{z}<1.6 \mathrm{~V} / \mathrm{nm}$, in helix structure 1 denoted by $(5,1)$ for $1.6 \mathrm{~V} /$ $\mathrm{nm}<E_{z}<2.8 \mathrm{~V} / \mathrm{nm}$, in helix structure 2 denoted by $(5,2)$ for $2.8 \mathrm{~V} /$ $\mathrm{nm}<E_{z}<6.2 \mathrm{~V} / \mathrm{nm}$, and a single chain inside denoted by 5 -shellchain structure $(5+1)$ as shown in Figure 6.

The transformation in the nanotubes for different $R$ shares one common feature, that is, as $E_{z}$ increases, the structure of the ice tube becomes helical and then a single chain along the axis inside. This is because compared to prism structure, the dipoles of water molecules in helix structure are more parallel to $E_{z}$. Thus, when $E_{z}$ is large enough, the free energy of the helix structure is lower than that of the prism structure. In addition, the dilating effect of water confined in nanotube mentioned above, in the direction perpendicular to the external field, makes it possible for a single chain along the axis to adapt inside. In nanotubes with $R=5.55 \AA$, increasing $E_{z}$ induces ice tube to transform from square prism to pentagonal prism, which can also be explained by the dilating effect of external field. The radius

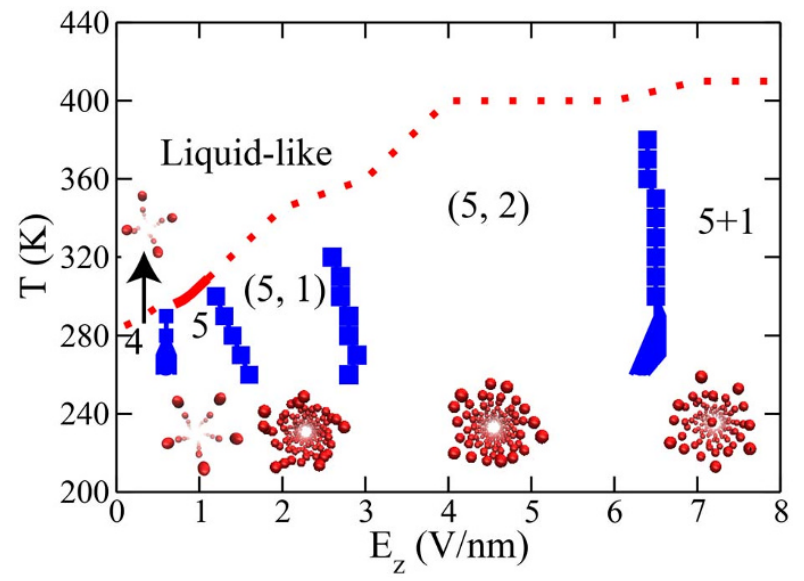

Figure $6 \mid$ The phase diagram of water confined within the SWCN for $R=5.55 \AA$ at atmospheric pressure. Filled squares denote different ice-ice phase boundaries. The shaded regions are the coexistence two ice phases. The dot line shows the estimated melting line between ice and liquid-like phase with continuous transition. The solid line is the estimated melting line between ice and liquid-like (discontinuous phase transition). We also present snapshots of structure of ices formed in the SWCN at $T=260 \mathrm{~K}$ in the inset (note that only oxygen atoms are shown here). 


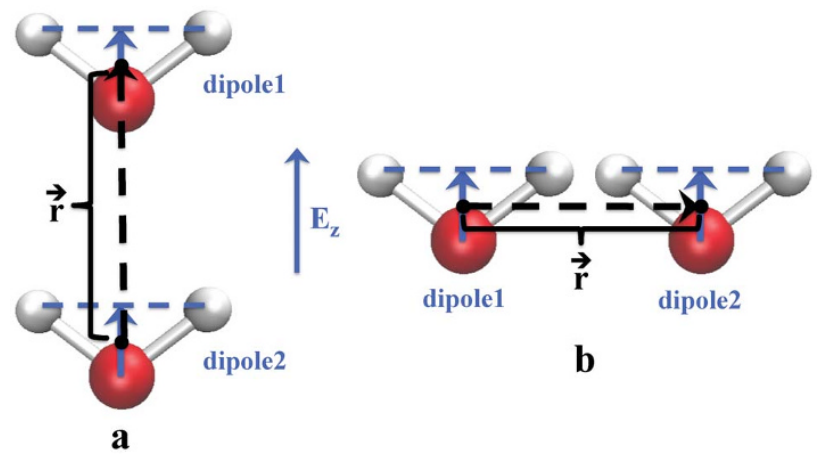

Figure 7 | Illustration of the orientations for two dipoles. (a) Two dipoles line up, and (b) two dipoles align parallel to each other. The attractive force is the strongest when two dipoles line up in the former case, while for repulsive force, it is a maximum at the same interdipole separation for two dipoles aligning parallel to each other in the latter case.

$R=5.55 \AA$ is somehow suitable for both square and pentagonal prism structures, and transformation between these two structures can also be induced by changing pressure ${ }^{5}$.

\section{Discussion}

According to the single-particle approximation theor $y^{35}$, if a dipole is considered as a particle immersed in an effective uniform electric field $E_{z}$, the distribution of polarization, $P(\cos \theta)$, follows the exponential form,

$$
P(\cos \theta) \sim \exp \left[\frac{E_{z} p \cos \theta}{k_{B} T}\right],
$$

where $p$ is the dipole moment, $\theta$ is the angle between the dipole and the uniaxial field $E_{z}$, and $k_{B}$ is the Boltzmann constant. This approximation is suitable for most polar liquids, but fails to reproduce the macroscopic permittivity of hydrogen-bonding systems such as bulk water ${ }^{36,37}$. It is believed that because the energy of hydrogen bond, $\sim 20 \mathrm{~kJ} / \mathrm{mol}$, is larger than the thermal energy and potential energy in external field, the coupling among the nearest molecules leads to its deviation from Eq. (2). Surprisingly, for confined water, the distribution follows the single-particle approximation to some extent [Figure 2]. This is possibly due to the fact that the restriction of hydrogen bonds on dipole rotation in confined water is weaker than that in bulk water.

For water in the SWCNT, the longitudinal electrostriction and cross-sectional dilation, can be explained by considering the interaction energy between dipoles. In a spherical coordinate system, a dipole, $\vec{P}$, can be described by $(p, \theta, \phi)$, where $p$ is the dipole moment, $\theta$ and $\phi$ are the polar angle and azimuthal angle ${ }^{38}$. The interaction energy between two dipoles, $\vec{P}_{1}\left(p_{1}, \theta_{1}, \phi_{1}\right), \vec{P}_{2}\left(p_{2}, \theta_{2}, \phi_{2}\right)$ (the direction connecting two dipoles, $\vec{r}$, is the polar axis in our study), can be calculated as

$U\left(r, \theta_{1}, \theta_{2}, \phi_{1}, \phi_{2}\right)=\frac{-p_{1} p_{2}\left(2 \cos \theta_{1} \cos \theta_{2}-\sin \theta_{1} \sin \theta_{2} \cos \left(\phi_{1}-\phi_{2}\right)\right)}{4 \pi \epsilon r^{3}}(3)$

Thus, the interaction force between two dipoles due to the presence of $E_{z}$ is $-\frac{\partial U\left(r, \theta_{1}, \theta_{2}, \phi_{1}, \phi_{2}\right)}{\partial r}$. According to Eq. 3, the interaction force between two dipoles along $E_{z}$ is attractive and strongest when the vector $(\vec{r})$ of two neighboring dipoles is parallel to $z$-axis in the case of $\theta_{1}=\theta_{2}=0$ [Figure 7(a)]. On the other hand, the interaction force between two dipoles along $E_{z}$ is repulsive and reaches a maximum when the vector $(\vec{r})$ of two neighboring dipoles is perpendicular to the $z$-axis in the case of $\theta_{1}=\theta_{2}=90^{\circ}$ [Figure 7(b)]. The presence of the uniaxial external field, dipoles tend to be parallel

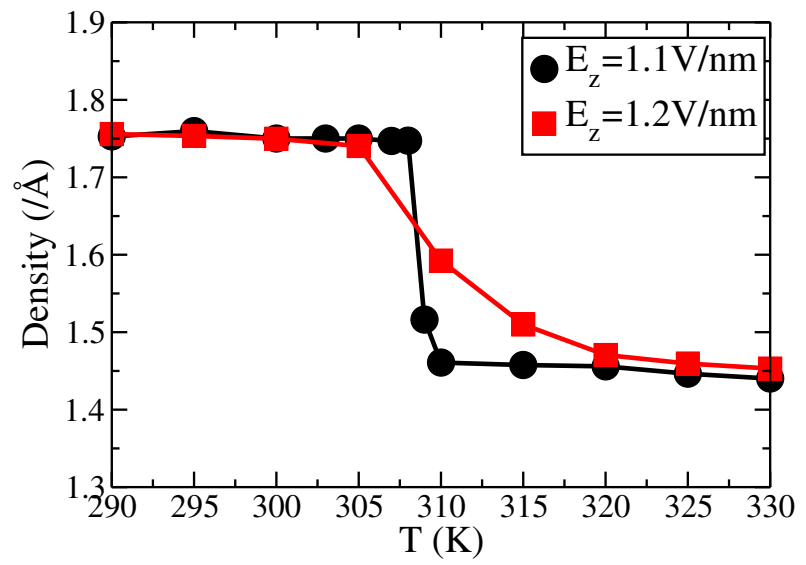

Figure $8 \mid$ The melting of ice at $E_{z}=1.1 \mathrm{~V} / \mathrm{nm}$ and $E_{z}=1.2 \mathrm{~V} / \mathrm{nm}$ field. The transformations from ice to liquid is a first-order phase transition for $E_{z}=1.1 \mathrm{~V} / \mathrm{nm}$, while it is a continuous transformation for $E_{z}=1.2 \mathrm{~V} / \mathrm{nm}$.

to the axis. This leads to water shrinking along $z$-direction and expanding across the nanotube.

Previous study by Koga et al. ${ }^{5}$ shows that water confined in the nanotube with $R=5.55 \AA$ exhibits a continuous phase transformation, from solid-like state to liquid-like state. In the presence of the uniaxial electrostatic field, we find that such transformation is a firstorder phase transition in the range of $0.6 \mathrm{~V} / \mathrm{nm}<E_{z}<1.1 \mathrm{~V} / \mathrm{nm}$, while it is a continuous transition for weaker field and stronger field. When the ordered ice is heated, the melting behavior is different. At $E_{z}<0.6 \mathrm{~V} / \mathrm{nm}$, the stable square prism at temperature $T<285 \mathrm{~K}$ gradually dilates and becomes a disordered square liquid (4 water molecules per layer on average) as temperature increases. This transition is continuous because the density fluctuation, $\left\langle\Delta \rho^{2}\right\rangle\left(=<\rho^{2}\right\rangle$ $-\left\langle\rho>^{2}\right)$, is continuous upon melting. However, at $0.6 \mathrm{~V} / \mathrm{nm}<E_{z}$ $<1.1 \mathrm{~V} / \mathrm{nm}$, upon heating, the system undergoes a discontinuous phase transition, from pentagonal prism structure at $T<300 \mathrm{~K}$ to square liquid at $T>300 \mathrm{~K}$. The discontinuous feature can be seen from the discontinuous change in the linear density as shown in Figure 8 for $E_{z}=1.1 \mathrm{~V} / \mathrm{nm}$. At larger external field, $1.1 \mathrm{~V} / \mathrm{nm}<$ $E_{z}<2.6 \mathrm{~V} / \mathrm{nm}$, upon heating, the melting of the stable $(5,1)$ helix ice becomes continuous again shown in Figure 8 at $E_{z}=1.2 \mathrm{~V} / \mathrm{nm}$. The system firstly transforms to a liquid-like $(5,1)$ helix structure before it finally melts to the square liquid. We note that both the orientation of dipoles and linear density undergo continuous change as the temperature increases. As external field further increases, the linear density continues to increase. The melting process of $(5,2)$ structure is similar to that of $(5,1)$ structure. Structure rearrangement does not occur for 5-shell-chain structure upon heating even in liquid state. Thus, water molecules still belong to shell-chain and linear density decreases slightly as temperature increases to $520 \mathrm{~K}$.

To summarize, we investigate the properties of water in SWCN in the presence of external field. We find that the external field induces the alignment of the dipoles of water molecules, directly leading to the longitudinal electrostriction and cross-sectional dilation of water in nanoscale. Water confined in SWCN also shows very rich phase behaviors, for instance, phase transformation from a prism structure to a helical one and then to a helical shell-chain as the magnitude of the electrostatic field increases. The stability of different phases is very much determined by the magnitude of external field. We determine the phase boundaries between each two phases and also propose a theory which well explains the stability of different phases. Our studies is important for the understanding of the properties of water confined in nanotubes, which can be of great importance for relevant industrial processes in the presence of external electrostatic field at nanocale. 


\begin{tabular}{|c|c|c|}
\hline Pair & $\epsilon(\mathrm{kJ} / \mathrm{mol})$ & $\sigma(\AA)$ \\
\hline $\begin{array}{l}\text { SWCN-O } \\
\text { SWCN-H }\end{array}$ & $\begin{array}{l}0.3876 \\
0.0\end{array}$ & $\begin{array}{l}3.262 \\
0.0\end{array}$ \\
\hline
\end{tabular}

\section{Methods}

Isothermal-isostress molecular dynamics (MD) simulations are performed on a model system of water in a single-walled carbon nanotube (SWCN). The system consists of 240 water molecules confined in a nanotube with radius $R$, which is simplified as smooth cylindrial wall. The system is subject to periodic boundary condition to the $z$-direction parallel (along the axis of the nanotube). All atoms in the computational cell are replicated in $z$-direction to form an infinite lattice. The uniaxial electrostatic field, $E_{z}$, if applied, is also along this direction.

The water-water interaction is described by the TIP4P water model ${ }^{39}$. The force field of the model SWCN is chosen as the integrated 12-6 Lennard-Jones potential integrating over the cylindrical area of the nanotube with the area density of the carbon atoms $\mathrm{s}^{5,40}$. Thus the interaction energy between a water molecule and the cylindrical wall is entirely determined by the distance of the oxygen site of the TIP4P water from the nanotube axis. The parameters of the model are shown in Table III. For all the simulations in this work, the particle-particle particle-mesh method $(\mathrm{PPPM})^{41}$ is used to caculate the long-range electrostatic interactions with a cutoff distance $r_{c}=8.5 \AA$.

The MD simulations are performed using LAMMPS software ${ }^{42}$ with the temperature $T$ and the axial-pressure $P_{z}$ controlled by the Nosé-Hoover algorithm. The pressure is fixed at $P_{z}=1 \mathrm{~atm}$ for all the thermodynamic states examined. Each simulation is performed for $60-120$ ns with time step of $0.5 \mathrm{fs}$.

1. Roux, B., Allen, T., Berneche, S. \& Im, W. Theoretical and computational models of biological ion channels. Q. Rev. Biophys. 37, 15-103 (2004).

2. Doyle, D. A., Morais, C. J., Pfuetzner, R. A. et al. The structure of the potassium channel: molecular basis of $K^{+}$conduction and selectivity. Science 280, 69-77 (1998).

3. Jähnert, S., Chávez, F. V., Schaumann, G. E. et al. Melting and freezing of water in cylindrical silica nanopores. Phys. Chem. Chem. Phys. 10, 6039-6051 (2008).

4. BellissentFunel, M. C., Lal, J. \& Bosio, L. Structural study of water confined in porous glass by neutron scattering. J. Chem. Phys. 98, 4246-4252 (1993).

5. Koga, K., Gao, G. T., Tanaka, H. \& Zeng, X. C. Formation of Ordered Ice Nanotubes Inside Carbon Nanotubes. Nature 412, 802-805 (2001).

6. Takaiwa, D., Hatano, I., Koga, K. \& Tanaka, H. Phase diagram of water in carbon nanotubes. Proc. Natl. Acad. Sci. USA 105, 3943 (2008).

7. Mattia, D. \& Calabrò, F. Explaining high flow rate of water in carbon nanotubes via solidliquid molecular interactions. Microfluid Nanofluid 13, 125-130 (2012).

8. Zhu, F. \& Schulten, K. Water and proton conduction through carbon nanotubes as models for biological channels. Biophys J. 85, 236-244 (2003).

9. Kalra, A., Garde, S. \& Hummer, G. Osmotic water transport through carbon nanotube membranes. Proc. Natl. Acad. Sci. USA 100, 10175-10180 (2003).

10. Hu, Z. J., Baralia, G., Bayot, V., Gohy, J. F. \& Jonas, A. M. Nanoscale control of polymer crystallization by nanoimprint lithography. Nano Lett. 5, 1738-1743 (2005).

11. Hamilton, B. D., Ha, J.-M., Hillmyer, M. A. \& Ward, M. D. Manipulating Crystal Growth and Polymorphism by Confinement in Nanoscale Crystallization Chambers. Acc. Chem. Res. 45, 414-423 (2012).

12. Rasaiah, J. C., Garde, S. \& Hummer, G. Water in nonpolar confinement: from nanotubes to proteins and beyond. Annu. Rev. Phys. Chem. 59, 713-740 (2008).

13. Yeh, I.-C. \& Berkowitz, M. L. Dielectric constant of water at high electric fields: molecular dynamics study. J. Chem. Phys. 110, 79357942 (1999).

14. Dzubiella, J. \& Hansen, J.-P. Electric-field-controlled water and ion permeation of a hydrophobic nanopore. J. Chem. Phys. 122, 234706-14 (2005).

15. Bratko, D., Daub, C. D. \& Luzar, A. Field-exposed water in a nanopore: liquid or vapour? Phys. Chem. Chem. Phys. 10, 6807-6813 (2008).

16. Vaitheeswaran, S., Rasaiah, J. C. \& Hummer, G. Electric field and temperature effects on water in the narrow nonpolar pores of carbon nanotubes. J. Chem. Phys. 121, 7955-7965 (2004).

17. Ranjan, V., Nardelli, M. B. \& Bernholc, J. Electric Field Induced Phase Transitions in Polymers: A Novel Mechanism for High Speed Energy Storage. Phys. Rev. Lett. 108, 087802-5 (2012).

18. Qiu, H. \& Guo, W. Electromelting of Confined Monolayer Ice. Phys. Rev. Lett. 110 195701-5 (2013)

19. Svishchev, I. M. \& Kusalik, P. G. Crystallization of liquid water in a molecular dynamics simulation. Phys. Rev. Lett. 73, 975-978 (1994).

20. Fu, Z., Luo, Y., Ma, J. \& Wei, G. Phase transition of nanotube-confined water driven by electric field. J. Chem. Phys. 134, 154507-6 (2011).

21. Kasinski, J. J., Gomez-Jahn, L. A., Faran, K. J., Gracewski, S. M. \& Dwayne Miller, R. J. Picosecond dynamics of surface electron transfer processes: Surface restricted transient grating studies of the $\mathrm{n} \mathrm{TiO} 2 / \mathrm{H} 2 \mathrm{O}$ interface. J. Chem. Phys. 90, 1253-1269 (1989).

22. Toney, M. F. et al. Voltage-dependent ordering of water molecules at an electrodeelectrolyte interface. Nature 368, 444-446 (1994).

23. Toney, M. F. et al. Distribution of water molecules at $\mathrm{Ag}(111) /$ electrolyte interface as studied with surface X-ray scattering. Surf. Sci. 335, 326-332 (1995).

24. Chu, Y. S., Lister, T. E., Cullen, W. G., You, H. \& Nagy, Z. Commensurate water monolayer at the RuO 2 (110)/water interface. Phys. Rev. Lett. 86, 3364-3367 (2001).

25. Ehre, D., Lavert, E., Lahav, M. \& Lubomirsky, I. Water freezes differently on positively and negatively charged surfaces of pyroelectric materials. Science 327 , 672-675 (2010).

26. Sancho, M., Partenskii, M. B., Dorman, V. \& Jordan, P. C. Extended dipolar chain model for ion channels: electrostriction effects and the translocational energy barrier. Biophysical journal 68, 427-433 (1995).

27. Bratko, D., Daub, C. D., Leung, K. \& Luzar, A. Effect of field direction on electrowetting in a nanopore. J. Am. Chem. Soc. 129, 2504-2510 (2007).

28. Daub, C. D., Bratko, D. \& Luzar, A. Nanoscale wetting under electric field from molecular simulations. Top. Curr. Chem. 307, 155-179 (2012).

29. Bratko, D., Daub, C. D. \& Luzar, A. Water-mediated ordering of nanoparticles in an electric field. Faraday discussions 141, 55-66 (2009).

30. Bratko, D., Daub, C. D. \& Luzar, A. Field-exposed water in a nanopore: liquid or vapour? Phys.Chem.Chem.Phys. 10, 6807-6813 (2008).

31. England, J. L., Park, S. \& Pande, V. S. Theory for an order-driven disruption of the liquid state in water. J. Chem. Phys. 128, 044503 (2008).

32. Kolesnikov, A. I. et al. Anomalously soft dynamics of water in a nanotube: a revelation of nanoscale confinement. Phys. Rev. Lett. 93, 035503 (2004).

33. Ghosh, S., Ramanathan, K. V. \& Sood, A. K. Water at nanoscale confined in singlewalled carbon nanotubes studied by NMR. EPL 65, 678 (2004).

34. Byl, O. et al. Unusual hydrogen bonding in water-filled carbon nanotubes. J. Am. Chem. Soc. 128, 12090-12097 (2006).

35. Onsager, L. Electric moments of molecules in liquids. J. Am. Chem. Soc. 58, 1486-1493 (1936).

36. Kirkwood, J. G. The dielectric polarization of polar liquids. J. Chem. Phys. 7, 911-919 (1939)

37. Danielewicz-Ferchmin, I. \& Ferchmin, A. R. Static permittivity of water revisited: $\epsilon$ in the electric field above $10^{8} \mathrm{Vm}^{-1}$ and in the temperature range $273 \leq T \leq$ 373K. Phys. Chem. Chem. Phys. 6, 1332-1339 (2004).

38. Israelachvili, J. N. Chapter 6, Van der Waals Forces, 107-130. Intermolecular and Surface Forces. (Sinapore: Elsevier, 2012).

39. Jorgensen, W. L., Chandrasekhar, J., Madura, J. D., Impey, R. W. \& Klein, M. L. Comparison of simple potential functions for simulating liquid water. J. Chem. Phys. 79, 926-935 (1983).

40. Steele, W. A. Interaction of Gases with Solid Surfaces (Pergamon, Oxford, 1974)

41. Hockney, R. W. \& Eastwood, J. W. Computer Simulation Using Particle (New York: Taylor \& Francis, 1989).

42. Plimpton, S. Fast parallel algorithms for short-range molecular dynamics. J. Comput. Phys. 117, 1-19 (1995)

\section{Acknowledgments}

We thank the National Science Foundation of China (NSFC) (Grant No.: 11290162/ A040106 and 11174006) and the National Basic Research Program of China (973 Program) (Grant No.: 2012CB921404) for financial supports. The work of K.K. was supported by a Grant- in-Aid for Scientific Research and the program for promoting the enhancement of research universities from MEXT, Japan.

\section{Author contributions}

G.S. and L.X. designed the work. G.S. and Y.H. performed the molecule dynamics simulation. Y.H., G.S., K.K. and L.X. analyzed the results. Y.H., G.S., K.K. and L.X. wrote the paper.

\section{Additional information}

Competing financial interests: The authors declare no competing financial interests.

How to cite this article: He, Y., Sun, G., Koga, K. \& Xu, L. Electrostatic field-exposed water in nanotube at constant axial pressure. Sci. Rep. 4, 6596; DOI:10.1038/srep06596 (2014).

This work is licensed under a Creative Commons Attribution-NonCommercialNoDerivs 4.0 International License. The images or other third party material in this article are included in the article's Creative Commons license, unless indicated otherwise in the credit line; if the material is not included under the Creative Commons license, users will need to obtain permission from the license holder in order to reproduce the material. To view a copy of this license, visit http:// creativecommons.org/licenses/by-nc-nd/4.0/ 